\title{
Triglyceride glycemic index as a biomarker for gestational diabetes mellitus: a systemic review and meta-analysis
}

\author{
Yusen Liu ${ }^{1, *}$, Ruiwen Chi ${ }^{1, *}$, Yujie Jiang ${ }^{2, *}$, Bicheng Chen ${ }^{1}$, Youli Chen ${ }^{3}$ and Zengrui Chen ${ }^{4}$ \\ ${ }^{1}$ Department of Cooperation and Communication, The First Clinical Medical College \& The First Affiliated Hospital of Wenzhou Medical University, \\ Wenzhou Medical University, Wenzhou, China \\ ${ }^{2}$ School of Ophthalmology \& Optometry, Wenzhou Medical University, Wenzhou, China \\ ${ }^{3}$ Wuhan Union Hospital, Tongji Medical College, Huazhong University of Science and Technology, Wuhan, China \\ ${ }^{4}$ Intensive Care Unit, The People's Hospital of Yuhuan, Yuhuan, China
}

Correspondence should be addressed to Z Chen or Y Chen: 848829511@qq.com or chenylpetri@gmail.com

*(Y Liu, R Chi and Y Jiang contributed equally to this work)

\begin{abstract}
Background: Triglyceride glycemic (TyG) index is a novel tool for assessing insulin resistance (IR). Recently, TyG index as a potential biomarker for gestational diabetes mellitus (GDM) has been studied, but its performance is yet inconclusive. Thus, we performed this systemic review and meta-analysis to evaluate the performance of TyG index in predicting GDM.

Methods: Studies published before March 1, 2021, with comparison of TyG index between GDM patients and healthy controls were retrieved from multiple databases (PubMed, Web of Science, The Cochrane Library, and Embase). The mean difference (MD) of TyG index in GDM patients and healthy controls was pooled using random-effect models. Results: Differentiation of TyG index between patients with GDM and controls showed significant results. Overall, there is a four-fold increase in TyG index in GDM patients compared with controls (MD: $0.22,95 \% \mathrm{Cl}: 0.07-0.36, P=0.003 ; R^{2}=71 \%, P=0.009$ ). In subgroup analyses according to gestational time, TyG index in the second trimester predicted GDM with low heterogeneity (MD: $0.26,95 \% \mathrm{Cl}: 0.15-0.37, P<0.001 ; R^{2}=0 \%$, $P=0.54$ ), while no such correlation was found in the first trimester.

Conclusion: TyG index, especially in the second trimester, could be a promising biomarker for predicting GDM.
\end{abstract}

Key Words

- gestational diabetes mellitus

- triglyceride glycemic index

- meta-analysis

\section{Introduction}

Gestational diabetes mellitus (GDM), the most common medical disorder in pregnancy, is defined as glucose intolerance resulting in hyperglycemia that begins or is first diagnosed during pregnancy (1). GDM is associated with adverse pregnancy outcomes such as cesarean section, birth injury, neonatal adiposity, and large size for gestational age of infants $(1,2)$. In addition, children born by mothers with GDM are more likely to develop obesity and impaired glucose tolerance (IGT), as well as type 2 diabetes in childhood or in early adulthood (3). Currently, the diagnosis for GDM is based on an oral glucose tolerance test (OGTT) conducted in 24-28 weeks of pregnancy (4), which could be a disadvantage for early prevention of adverse outcomes of GDM: metabolic disorders affecting fetal growth could develop before the diagnosis of gestational diabetes mellitus (GDM) in https://ec.bioscientifica.com

https://doi.org/10.1530/EC-21-0234 (c) 2021 The authors Published by Bioscientifica Ltd

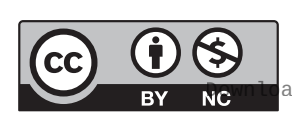

This work is licensed under a Creative Commons Attribution-NonCommercial 4.0 International License. ded from Bioscientifica.com at 04/26/2023 09:43:28AM 
elderly and obese women (5), and the sensitivity of OGTT is not satisfactory in early pregnancy (6). Thus, a new early screening method for GDM is warranted, especially in high-risk groups.

Available evidence suggests that the main pathogenesis of GDM is related to decreased insulin secretion and increased insulin resistance (IR) during pregnancy (7). Thus, identifying IR status could be beneficial for diagnosing GDM. However, current assessment methods, hyperinsulinemic-euglycemic clamp (HEC) and homeostatic model assessment for insulin resistance (HOMA-IR), are inconvenient to use in clinical settings because of excessive time and resources required. HEC requires fasting, taking blood samples frequently, and i.v. infusion of insulin and glucose, while HOMA-IR requires overnight fasting and plasma insulin measurement several times. At present, though many types of biomarkers have been tested individually or in predictive models for GDM as well, such as adiponectin, sex hormone-binding globulin, and C-reactive protein $(8,9,10)$, these parameters are also not available in most clinics. Therefore, identifying feasible biomarkers is warranted for predicting GDM in early pregnancy.

Triglyceride glycemic $(\mathrm{TyG}, \mathrm{TyG}$ index $=(\mathrm{ln}$ (fasting triglycerides $(\mathrm{TG})(\mathrm{mg} / \mathrm{dL}) \times$ fasting glucose $(\mathrm{mg} / \mathrm{dL}) / 2))$ ) index as a biomarker for IR, and GDM is gaining increasing attention. Studies have shown that TyG index is highly sensitive in identifying IR, especially for adults and obese adolescents $(11,12,13,14,15)$. In several previous studies, TyG index out-performed HOMO-IR in the Brazilian population (16) and the Korean population (17) in predicting the risk of diabetes. Measurement of TyG is also cheap and convenient (18), which is more accessible in outpatient settings, and could be a screening tool for large populations.

However, results from existing studies on the performance of TyG index in predicting GDM are inconsistent. Some studies showed a correlation between TyG index and GDM $(19,20,21,22)$, whereas others suggested there was no such correlation (23). These studies are limited in statistical power because of small study samples. Thus, we performed a systemic review and pooled these results using a meta-analytical approach. This study is critical for improving clinical practices regarding early detection and interventions to prevent GDM and related pregnancy complications in a cheap and convenient way, which could be particularly significant for improving maternal prenatal care in low-income areas (24).

\section{Methods}

\section{Search strategy}

The Preferred Reporting Items for Systematic Reviews and Meta-Analyses (PRISMA) (25) reporting guideline was cited to conduct the study. Four databases, PubMed, Web of Science, The Cochrane Library, and Embase, were searched in this study up to March 1, 2020. Keywords used for comprehensive literature search were 'Gestational Diabetes Mellitus', 'PregnancyInduced Diabetes', 'Gestational Diabetes', and 'triglyceride-glucose index'. There was no language filter applied. A detailed search strategy is presented in the Supplementary Materials.

\section{Eligibility criteria and study selection}

Full texts of studies were included in this meta-analysis if they: (i) were cross-sectional study, case-control study, cohort study, randomized controlled trial, nonrandomized controlled trial, or prospective study conducted in pregnant women; (ii) compared TyG index between individuals with GDM and healthy controls; (iii) reported diagnosis for GDM; (iv) reported sample size. Exclusion criteria were (i) articles with incomplete or unmanageable data; (ii) the definition of TyG index and GDM is not clear or the calculation method of TyG index was not mentioned; (iii) duplicate studies; (iv) case reports, comments, conferences, reviews, academic dissertations, and book chapters.

After removing duplicates, the titles and abstracts of the papers were independently screened by two researchers (Y S Liu and R W Chi) to determine whether the study met the inclusion criteria. The eligibility of the full-text reports was then assessed by two researchers (Y S Liu and R W Chi) independently. Any divergence was settled by discussion or submitted to a third examiner (Y J Jiang) for arbitration.

\section{Data extraction}

Retrieved information consisted of bibliographic information and study-specific details, including study location and year of study conduction, patient characteristics such as age, sex, and BMI. Fasting plasma glucose, fasting triglycerides (TG), and TyG index were also extracted for analysis. Any disagreements were discussed and resolved through consensus.

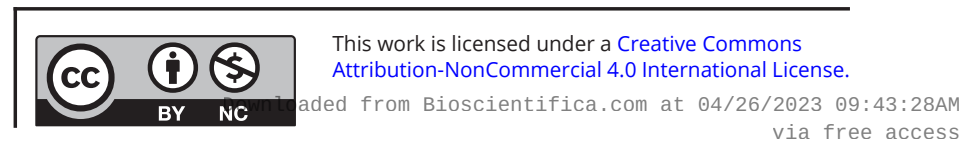




\section{Quality assessment}

The Newcastle-Ottawa Scale (NOS) was used for assessing the quality of each study. Briefly, this system evaluates studies based on participant selection (4 points), comparability of study groups ( 2 points), and assessment of outcome or exposure ( 3 points) (26). A score $>7$ indicates high quality, and a score $<4$ indicates low quality.

\section{Statistical analysis}

Continuous variables were expressed as mean (S.D.). Mean difference (MD) was used to evaluate the difference in TyG index between patients with GDM and controls. The randomeffect model was used. $I^{2}$ statistic was used to examine the heterogeneity of included studies, assigning low, moderate, and high heterogeneity to corresponding $I^{2}$ values of 25,50 , and $75 \%$. Additionally, subgroup analysis was performed regarding the time of pregnancy and study location. The publication bias was assessed by visual inspection of funnel charts and Egger's test, and sensitivity analysis was carried out to determine the robustness of the results.

The data analysis was performed using the Review Manager (version 5.4 for Windows; the Cochrane Collaboration, Oxford, United Kingdom) and Stata 16.1 (StataCorp. 2019. Stata Statistical Software: Release 16.
StataCorp LLC). $P$ values $<0.05$ were considered to be statistically significant.

\section{Results}

Our initial search identified 824 articles, which was reduced to 665 when duplicates were excluded. Six hundred fifty-seven articles were excluded based on title and abstract. From the remaining articles, we excluded four publications for insufficient data, and finally identified four eligible articles (Fig. 1).

\section{Characteristics of included studies and study quality}

Four prospective cohort studies were included in the metaanalysis, involving 1610 participants. Participants in each study ranged from 140 to 954 . Two were conducted in Mexico, and the rest were in China and Iran. The average age of the participants ranged from 24.23 to 30.4 years. All studies had clear recruitment exclusion criteria, and two studies reported that eligible participants accounted for 81 (22) and 97\% (19) of all enrolled participants, respectively. Sánchez-García and Rodríguez-Gutiérrez (21) collected blood samples during the second trimester

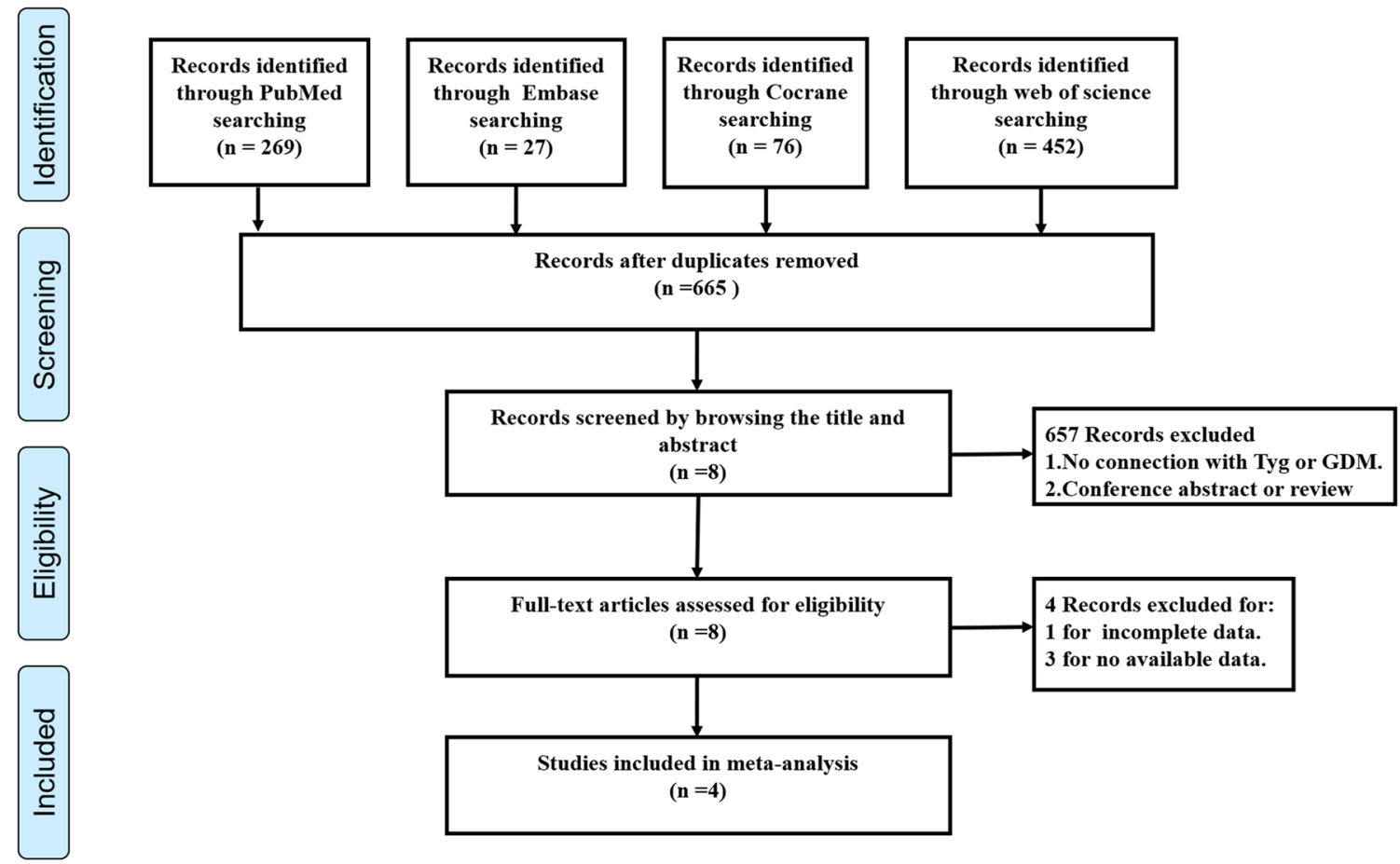

Figure 1

Study identification and selection. 


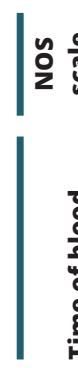

离
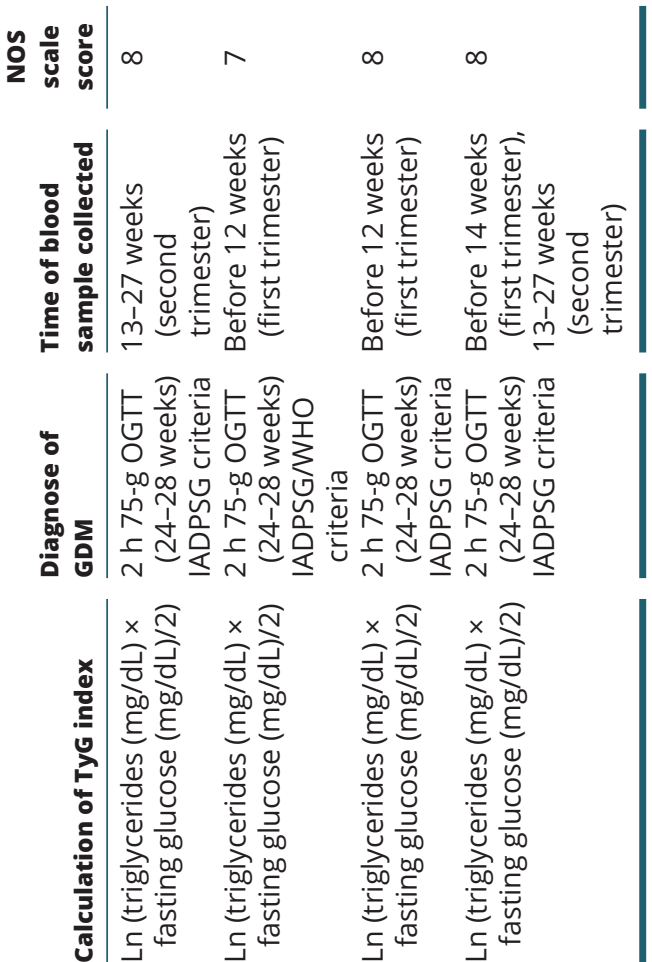

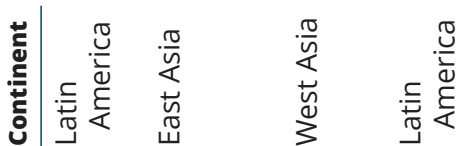



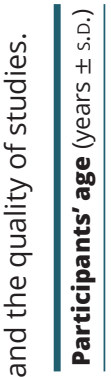

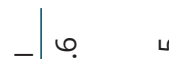

อ

仓े

N

$\sum \mid$

讨

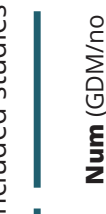

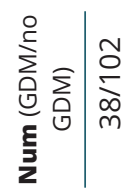

$\stackrel{\infty}{\stackrel{\infty}{v}}$

กำ

$+1$

จ่

$\stackrel{0}{\circ}$
$\dot{+}$
+1
$\stackrel{+}{\circ}$
$m$

$\dot{0}$

ס

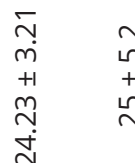

เ

m

๙

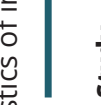

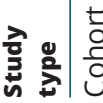

$\frac{5}{0}$

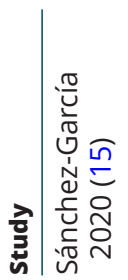

엃

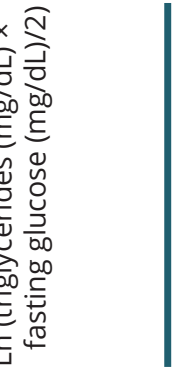

ب

in

ก

$\stackrel{\substack{4 \\+1}}{+1}$



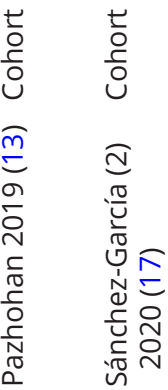

(13-27 weeks), Liu et al. (22) and Pazhohan et al. (19) collected blood samples during the first trimester ( $<12$ weeks), and another study by Sánchez-García \& Rodríguez-Gutiérrez (23) collected blood samples during both the first and second trimesters. Detailed study characteristics are shown in Table 1. Detailed assessment of the quality of studies is presented in Supplementary Table 1 (see section on supplementary materials given at the end of this article). Study quality ranged from 7 to 8 points out of 9 , indicating that the methodological quality of the literature included in this study was high.

\section{TyG index and GDM}

All four studies reported outcomes of interest. The pooled TyG index of GDM patients was significantly higher than that of the control group without GDM (MD: 0.22 , 95\% CI: 0.07 to $0.36, P=0.003$ ) with a high level of heterogeneity $\left(I^{2}=71 \%, P=0.009\right)$ (Fig. 2A).

Subgroup analysis regarding the time of pregnancy (first and second trimester) was conducted. No difference in TyG index was found between GDM patients and healthy controls in first trimester (MD: 0.22, 95\% CI: -0.07 to 0.51 , $P=0.14, I^{2}=84 \%$ ) (Fig. 2B), while mid-gestation (second trimester) maternal TyG index is significantly higher in GDM patients (MD: $0.26,95 \%$ CI: 0.15 to $0.37, P<0.001$ ). When subgroup analysis only included studies measuring TyG at second trimester, the level of heterogeneity was low $\left(I^{2}=0 \%\right)$ (Fig. 2C).

When subgroup analysis was done according to study locations (Latin and Asia), the predictive value of TyG for GDM was non-significant in Asians (MD: 0.45, 95\% CI: -0.10 to $1.01, P=0.11$ ) or Mexicans (MD: $0.16,95 \%$ CI: -0.02 to $-0.34, P=0.08)$. However, the heterogeneity decreased in the Asian group $\left(I^{2}=45 \%, P=0.18\right)$ (Fig. 2D).

The results were robust according to sensitivity analysis (Fig. 3). We used Egger's test for the detection of publication bias (Fig. 4), and no significant bias was detected in included studies $(P>|\mathrm{t}|=0.128)$.

\section{Discussion}

TyG index is one of the emerging biomarkers for IR identification and GDM prediction, which is cheap and convenient to practice, and could predict GDM earlier than the OGTT diagnoses (usually 24-28 weeks), which enables physicians to take early actions to prevent GDM and to protect patients from the risk of pregnancy complications. Quantitatively analyzing the possible 
A

\begin{tabular}{l} 
Study or Subgroup \\
\hline Liu 2020 \\
Pazhohan 2019 \\
Sánchez-García (2) 2020 \\
Sánchez-García (2) 2020 \\
Sánchez-García 2020
\end{tabular}

Experimental Control

Mean Difference

Mean Difference

Mean SD Total Mean SD Total Weight IV, Random, $95 \% \mathrm{CI}$

$\begin{array}{rrrrrrrr}8.3 & 0.4 & 66 & 8 & 0.4 & 286 & 28.1 \% & 0.30[0.19,0.41]\end{array}$

$\begin{array}{llllllll}9.31 & 6.13 & 176 & 8.34 & 5.04 & 778 & 2.0 \% & 0.97[-0.00,1.94]\end{array}$

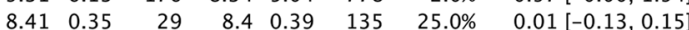

$\begin{array}{llllllll}9.01 & 0.3 & 29 & 8.73 & 0.34 & 135 & 26.7 \% & 0.28[0.16,0.40]\end{array}$

Sánchez-García 2020

$\begin{array}{lllllll}4.88 & 0.7 & 38 & 4.68 & 0.19 & 102 & 18.2 \%\end{array}$

Total $(95 \% \mathrm{Cl})$

338

$1436 \quad 100.0 \%$

Heterogeneity: $\mathrm{Tau}^{2}=0.02 ; \mathrm{Chi}^{2}=13.59, \mathrm{df}=4(\mathrm{P}=0.009) ; \mathrm{I}^{2}=71 \%$

$0.20[-0.03,0.43]$ Test for overall effect: $Z=2.98(P=0.003)$

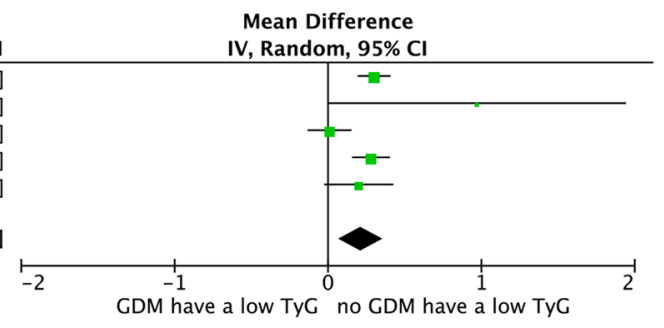

B

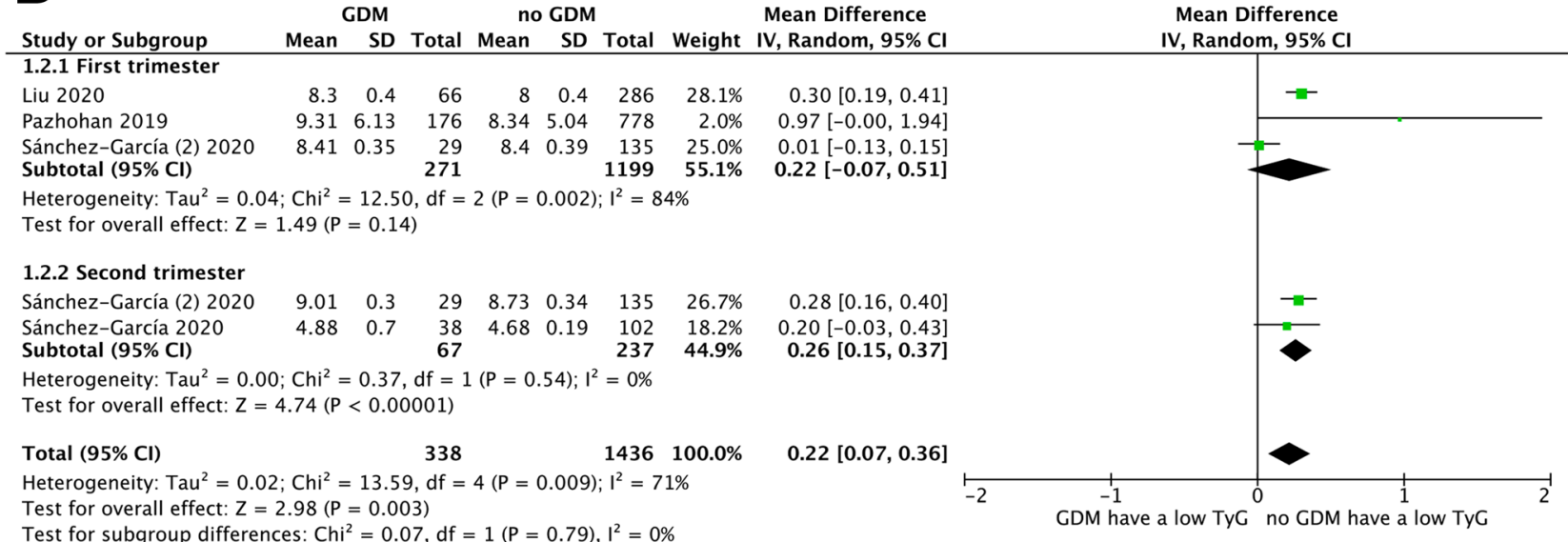

Test for subgroup differences: $\mathrm{Chi}^{2}=0.07, \mathrm{df}=1(\mathrm{P}=0.79), \mathrm{I}^{2}=0 \%$

C

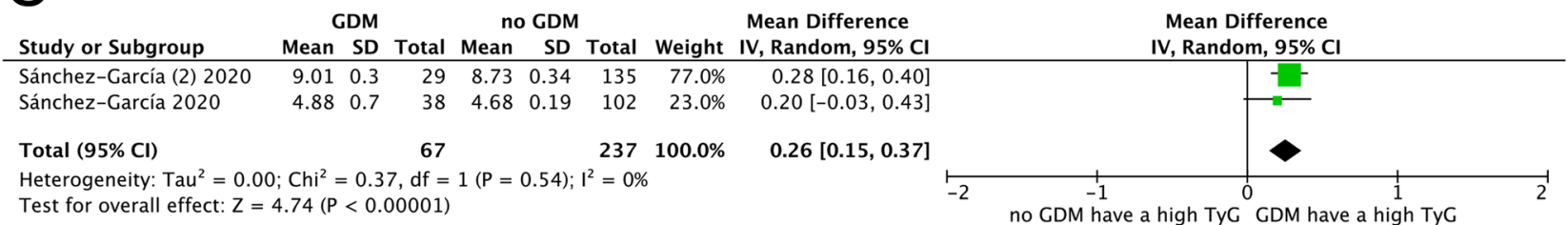

D

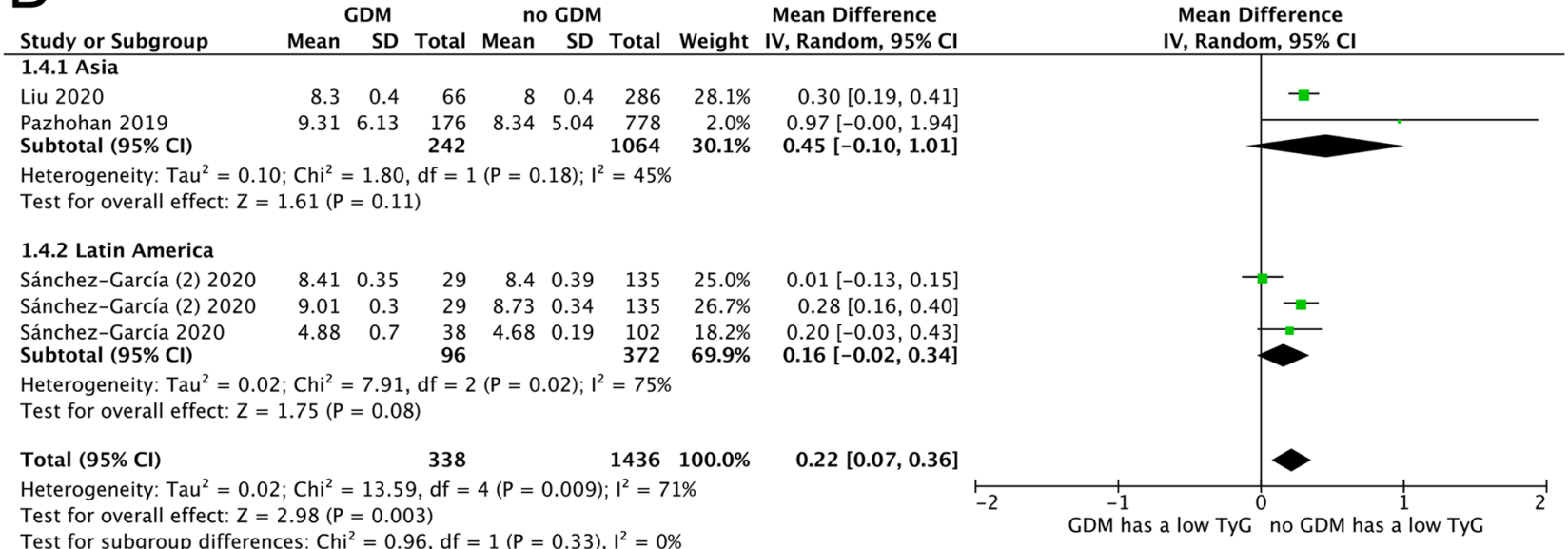

\section{Figure 2}

Forest plots. (A) Forest plot of difference of TyG index between GDM patients and healthy controls. (B) Forest plot of difference of TyG index in the first trimester and second trimester between GDM patients and healthy controls. (C) Forest plot of difference of TyG index only in the second trimester. (D) Forest plot of difference of TyG index based on study locations.

https://ec.bioscientifica.com https://doi.org/10.1530/EC-21-0234 (c) 2021 The authors Published by Bioscientifica Ltd

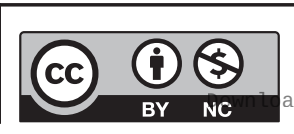

This work is licensed under a Creative Commons Attribution-NonCommercial 4.0 International License. ded from Bioscientifica.com at 04/26/2023 09:43:28AM 
relationship between outcomes of GDM and TyG index levels using a meta-analytical approach could aid clinical decisions. The strengths of this study are the rigorous methods used for the comprehensive literature search, and strict study inclusion criteria. Source heterogeneity among studies were extensively studied using subgroup analysis and sensitivity analysis.

Our study suggests that high TyG index may be a potential predictor for GDM (MD: 0.22, 95\% CI: 0.07 to $0.36, P=0.003$ ). A recent study by Song et al. (27) has concluded that women with the highest TyG category are at higher risk of subsequent GDM (OR: 2.52, 95\% CI: 1.33-4.67; I2 $=65 \%, P=0.004$ ), especially in Asia (OR: 3.30 , 95\% CI: $1.50-7.28, P=0.003)$, using data measured before pregnancy and during the first trimester. In our study, continuous data of TyG were preserved in the analyses, using effect sizes based on MDs instead of ORs. Different study focus, search strategy, and inclusion criteria contributed to inconsistency of results between the two studies. Future studies should complement each other and provide a wholesome, theoretical basis for subsequent researchers to continue exploring the predictive value of TyG. However, the heterogeneity is high across studies $\left(I^{2}=71 \%, P=0.009\right)$. To identify sources of heterogeneity, we carried out subgroup analyses according to the time of measurement and study location.

When subgroup analysis was conducted regarding the time of TyG measurement, heterogeneity among studies was significantly reduced. A three-fold increase in TyG index in GDM patients was found compared with healthy controls (MD: $0.26,95 \%$ CI: 0.15 to $0.37, P<0.001$; $\left.I^{2}=0 \%, P=0.54\right)$. However, TyG index's performance in the first trimester remains inconclusive (MD: 0.22, 95\% CI: -0.07 to $\left.0.51, P=0.14 ; I^{2}=84 \%, P=0.002\right)$. According

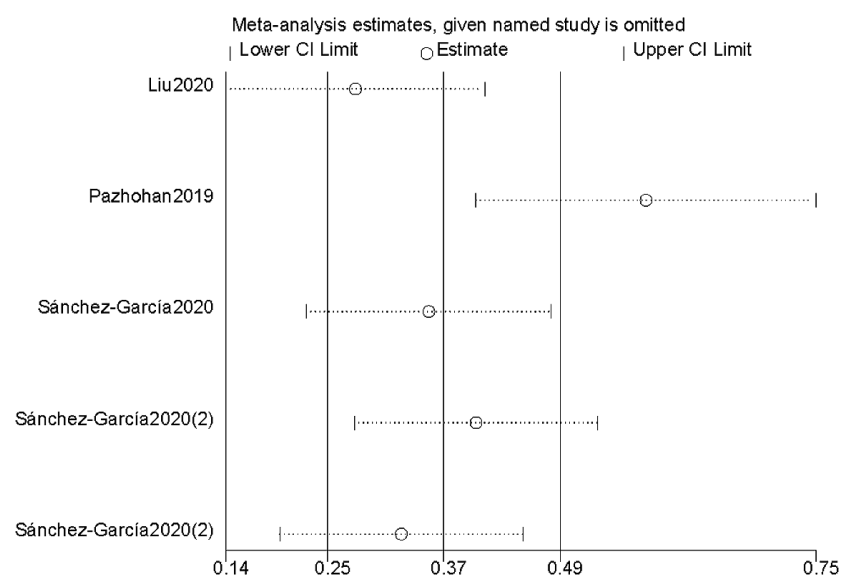

Figure 3

Sensitivity analysis.

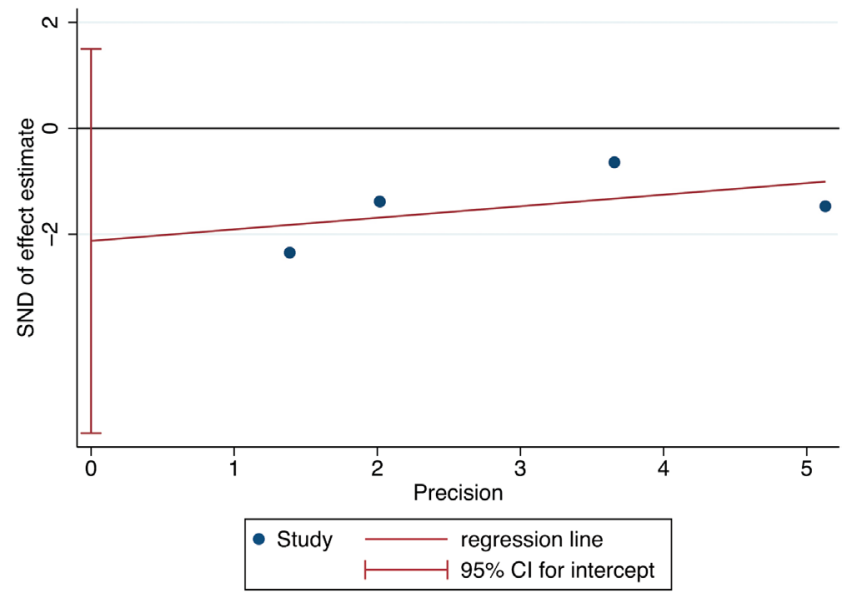

Figure 4

Egger's test for the publication bias.

to a previous cross-sectional study conducted by Wiznitzer et al. (28), the levels of TG in a healthy pregnant woman are fluctuant throughout the pregnancy to meet the needs of the fetus, which decreases during the first 2 months of the pregnancy and gradually increased in the next 7 months. Thus, the comparison of TyG index calculated before or around 2 months could be more sensitive. Secondly, individual difference of participants could contribute to the heterogeneity in TyG levels. For example, racial difference in physiology should be considered. A previous study (29) found the cut-off values of TyG index for identifying IR ranged from 8.45 to $8.65,8.35$ to 8.55 , and 8.15 to 8.35 in Mexican American (non-Hispanic white), Korean, and non-Hispanic black adolescents, respectively, which showed that reference values of TyG index could be different in individuals with different racial backgrounds. Furthermore, though all from Mexico, the TyG level of two separate cohorts from the same center showed a significant difference, which could be due to technical differences in implementing tests. In our study, though TyG index predicted GDM overall, it showed nonsignificant predicative values in either subgroup divided by study location (Latin America and Asia).

Heterogeneity introduced by individual studies was inspected using sensitivity analysis. Significantly higher level of TyG was noted in the cohort reported by Pazhohan (19), and the lowest level of TyG in Mexico was reported by Sánchez-García (21). However, the results were robust according to sensitivity analysis. What would also contribute to the heterogeneity is the management of confounding factors when enrolling patients. SánchezGarcía et al. $(21,23)$ adjusted for age, parity, BMI, systolic blood pressure, gestational age at OGTT, gestational weight

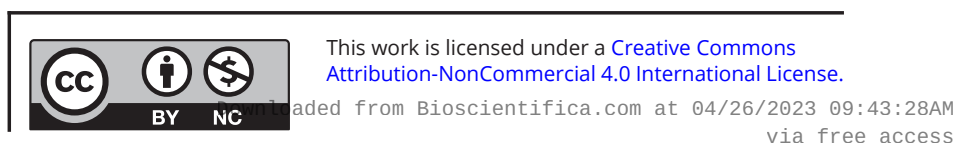


gain, and etc. However, in Pazhohan 2019 (19), significant differences existed between GDM group and control group in participant's age, history of diabetes in the first-degree family, and first trimester-BMI. Similarly, participants in Liu 2020 (22) also owed obvious difference in BMI, physical activity, etc.

Although this study provides promising results, there are limitations, which warrant future research. A major limitation of this study is the small number of existing research, which may introduce small sample bias, and restrict some of the analyses such as meta-regression to control for confounders among studies. Secondly, most of the included studies were cohort studies; thus, a causal relationship between TyG and GDM could not be proved. Additionally, Pazhohan et al. (19) and Liu et al. (22) did not control for confounders adequately, and owing to ethical reasons, individual-level data were not available from these studies, preventing us from doing further adjustments. Lastly, due to the fact that included studies are just from Mexico, Iran, and China, the generalizability of these findings could be limited. To confirm the relationship between TyG index and GDM, and investigate its potential mechanisms, more large-size, randomized controlled trials are needed across different regions. Moreover, future research should carry out TyG index measurement at different time points during the pregnancy to optimize its predicative value of GDM.

In conclusion, considering that it is readily available, cheap, and convenient, TyG index is a promising biomarker for predicting GDM, especially in the second trimester. This could potentially improve the current practice for better population coverage and earlier diagnostic time.

\section{Supplementary materials}

This is linked to the online version of the paper at https://doi.org/10.1530/ EC-21-0234.

\section{Declaration of interest}

The authors declare that there is no conflict of interest that could be perceived as prejudicing the impartiality of the research reported.

\section{Funding}

This work was supported by the National Training Program of Innovation and Entrepreneurship for Undergraduates (No. 201910343014).

\section{References}

1 Saravanan P, Diabetes in Pregnancy Working Group, Maternal Medicine Clinical Study Group \& Royal College of Obstetricians and
Gynaecologists, UK. Gestational diabetes: opportunities for improving maternal and child health. Lancet: Diabetes and Endocrinology 20208 793-800. (https://doi.org/10.1016/S2213-8587(20)30161-3)

2 Catalano PM, McIntyre HD, Cruickshank JK, McCance DR, Dyer AR, Metzger BE, Lowe LP, Trimble ER, Coustan DR, Hadden DR, et al. The hyperglycemia and adverse pregnancy outcome study: associations of GDM and obesity with pregnancy outcomes. Diabetes Care 201235 780-786. (https://doi.org/10.2337/dc11-1790)

3 Lowe Jr WL, Scholtens DM, Kuang A, Linder B, Lawrence JM, Lebenthal Y, McCance D, Hamilton J, Nodzenski M, Talbot O, et al. Hyperglycemia and adverse pregnancy outcome follow-up study (HAPO FUS): maternal gestational diabetes mellitus and childhood glucose metabolism. Diabetes Care 201942 372-380. (https://doi. org/10.2337/dc18-1646)

4 International Association of Diabetes and Pregnancy Study Groups Consensus Panel, Metzger BE, Gabbe SG, Persson B, Buchanan TA, Catalano PA, Damm P, Dyer AR, Leiva Ad, Hod M, et al. International association of diabetes and pregnancy study groups recommendations on the diagnosis and classification of hyperglycemia in pregnancy. Diabetes Care 201033 676-682. (https://doi.org/10.2337/dc09-1848)

5 Kim W, Park SK \& Kim YL. Gestational diabetes mellitus diagnosed at 24 to 28 weeks of gestation in older and obese women: is it too late? PLoS ONE 201914 e0225955. (https://doi.org/10.1371/journal. pone.0225955)

6 Lekva T, Godang K, Michelsen AE, Qvigstad E, Normann KR, Norwitz ER, Aukrust P, Henriksen T, Bollerslev J, Roland MCP, et al. Prediction of gestational diabetes mellitus and pre-diabetes 5 years postpartum using $75 \mathrm{~g}$ oral glucose tolerance test at 14-16 weeks' gestation. Scientific Reports 20188 13392. (https://doi.org/10.1038/ s41598-018-31614-z)

7 Bowers K, Yeung E, Williams MA, Qi L, Tobias DK, Hu FB \& Zhang C. A prospective study of prepregnancy dietary iron intake and risk for gestational diabetes mellitus. Diabetes Care 201134 1557-1563. (https://doi.org/10.2337/dc11-0134)

8 Wolf M, Sandler L, Hsu K, Vossen-Smirnakis K, Ecker JL \& Thadhani R. First-trimester C-reactive protein and subsequent gestational diabetes. Diabetes Care 200326 819-824. (https://doi.org/10.2337/ diacare.26.3.819)

9 Smirnakis KV, Plati A, Wolf M, Thadhani R \& Ecker JL. Predicting gestational diabetes: choosing the optimal early serum marker. American Journal of Obstetrics and Gynecology 2007196 410.e1-410.e6; discussion 410.e6-410.e7. (https://doi.org/10.1016/j.ajog.2006.12.011)

10 Savvidou M, Nelson SM, Makgoba M, Messow CM, Sattar N \& Nicolaides K. First-trimester prediction of gestational diabetes mellitus: examining the potential of combining maternal characteristics and laboratory measures. Diabetes 201059 3017-3022. (https://doi.org/10.2337/db10-0688)

11 Unger G, Benozzi SF, Perruzza F \& Pennacchiotti GL. Triglycerides and glucose index: a useful indicator of insulin resistance. Endocrinologia y Nutricion 201461 533-540. (https://doi.org/10.1016/j. endonu.2014.06.009)

12 Du T, Yuan G, Zhang M, Zhou X, Sun X \& Yu X. Clinical usefulness of lipid ratios, visceral adiposity indicators, and the triglycerides and glucose index as risk markers of insulin resistance. Cardiovascular Diabetology 201413 146. (https://doi.org/10.1186/s12933-014-0146-3)

13 Mohd Nor NS, Lee S, Bacha F, Tfayli H \& Arslanian S. Triglyceride glucose index as a surrogate measure of insulin sensitivity in obese adolescents with normoglycemia, prediabetes, and type 2 diabetes mellitus: comparison with the hyperinsulinemic-euglycemic clamp. Pediatric Diabetes 201617 458-465. (https://doi.org/10.1111/ pedi.12303)

14 Irace C, Carallo C, Scavelli FB, De Franceschi MS, Esposito T, Tripolino C \& Gnasso A. Markers of insulin resistance and carotid atherosclerosis. A comparison of the homeostasis model assessment and triglyceride glucose index. International Journal of Clinical Practice 201367 665-672. (https://doi.org/10.1111/ijcp.12124) https://ec.bioscientifica.com https://doi.org/10.1530/EC-21-0234 (c) 2021 The authors Published by Bioscientifica Ltd
This work is licensed under a Creative Commons Attribution-NonCommercial 4.0 International License. ded from Bioscientifica, com at 04/26/2023 09:43:28AM 
15 Ozcimen EE, Uckuyu A, Ciftci FC, Yanik FF \& Bakar C. Diagnosis of gestational diabetes mellitus by use of the homeostasis model assessment-insulin resistance index in the first trimester. Gynecological Endocrinology 200824 224-229. (https://doi. org/10.1080/09513590801948416)

16 Vasques AC, Novaes FS, de Oliveira Mda S, Souza JR, Yamanaka A, Pareja JC, Tambascia MA, Saad MJ \& Geloneze B. TyG index performs better than HOMA in a Brazilian population: a hyperglycemic clamp validated study. Diabetes Research and Clinical Practice 201193 e98-e100. (https://doi.org/10.1016/j.diabres.2011.05.030)

17 Lee SH, Kwon HS, Park YM, Ha HS, Jeong SH, Yang HK, Lee JH, Yim HW, Kang MI, Lee WC, et al. Predicting the development of diabetes using the product of triglycerides and glucose: the Chungju Metabolic Disease Cohort (CMC) study. PLoS ONE 20149 e90430. (https://doi.org/10.1371/journal.pone.0090430)

18 Gasevic D, Frohlich J, Mancini GB \& Lear SA. The association between triglyceride to high-density-lipoprotein cholesterol ratio and insulin resistance in a multiethnic primary prevention cohort. Metabolism: Clinical and Experimental 201261 583-589. (https://doi.org/10.1016/j. metabol.2011.09.009)

19 Pazhohan A, Rezaee Moradali M \& Pazhohan N. Association of firsttrimester maternal lipid profiles and triglyceride-glucose index with the risk of gestational diabetes mellitus and large for gestational age newborn. Journal of Maternal-Fetal and Neonatal Medicine 201932 1167-1175. (https://doi.org/10.1080/14767058.2017.1402876)

20 Kim JA, Kim J, Roh E, Hong SH, Lee YB, Baik SH, Choi KM, Noh E, Hwang SY, Cho GJ, et al. Triglyceride and glucose index and the risk of gestational diabetes mellitus: a nationwide population-based cohort study. Diabetes Research and Clinical Practice 2021171 108533. (https:// doi.org/10.1016/j.diabres.2020.108533)

21 Sánchez-García A, Rodríguez-Gutiérrez R, Saldívar-Rodríguez D, Guzmán-López A, Castillo-Castro C, Mancillas-Adame L, SantosSantillana K, González-Nava V \& González-González JG. Diagnostic accuracy of the triglyceride-glucose index for gestational diabetes screening: a practical approach. Gynecological Endocrinology 202036 1112-1115. (https://doi.org/10.1080/09513590.2020.1742687)

22 Liu M, Chen Y \& Chen D. Association between transthyretin concentrations and gestational diabetes mellitus in Chinese women.
Archives of Gynecology and Obstetrics 2020302 329-335. (https://doi. org/10.1007/s00404-020-05599-y)

23 Sánchez-García A, Rodríguez-Gutiérrez R, Saldívar-Rodríguez D, Guzmán-López A, Mancillas-Adame L, González-Nava V, SantosSantillana K \& González-González JG. Early triglyceride and glucose index as a risk marker for gestational diabetes mellitus. International Journal of Gynaecology and Obstetrics 2020151 117-123. (https://doi. org/10.1002/ijgo.13311)

24 Sánchez-García A, Rodríguez-Gutiérrez R, Saldívar-Rodríguez D, Guzmán-López A, Castillo-Castro C, Mancillas-Adame L, SantosSantillana K, González-Nava V \& González-González JG. Diagnostic accuracy of the triglyceride-glucose index for gestational diabetes screening: a practical approach. Gynecological Endocrinology 202036 1112-1115. (https://doi.org/10.1080/09513590.2020.1742687)

25 McInnes MDF, Moher D, Thombs BD, McGrath TA, Bossuyt PM, and the PRISMA-DTA Group, Clifford T, Cohen JF, Deeks JJ, Gatsonis C, et al. Preferred reporting items for a systematic review and meta-analysis of diagnostic test accuracy studies: the PRISMADTA statement. JAMA 2018319 388-396. (https://doi.org/10.1001/ jama.2017.19163)

26 Wells G, Shea B, O'Connell D, Peterson J, Welch V, Losos M \& Tugwell P. The Newcastle-Ottawa Scale (NOS) for assessing the quality of nonrandomised studies in meta-analyses. Ottawa, Ontario, Canada: University of Ottawa. (available at: http://www.ohri.ca/programs/ clinical_epidemiology/oxford.asp)

27 Song T, Su G, Chi Y, Wu T, Xu Y \& Chen C. Triglyceride-glucose index predicts the risk of gestational diabetes mellitus: a systematic review and meta-analysis. Gynecological Endocrinology 2021 [epub]. (https:// doi.org/10.1080/09513590.2021.1940932)

28 Wiznitzer A, Mayer A, Novack V, Sheiner E, Gilutz H, Malhotra A $\&$ Novack L. Association of lipid levels during gestation with preeclampsia and gestational diabetes mellitus: a population-based study. American Journal of Obstetrics and Gynecology 2009201482. e1-482.e8. (https://doi.org/10.1016/j.ajog.2009.05.032)

29 Moon S, Park JS \& Ahn Y. The cut-off values of triglycerides and glucose index for metabolic syndrome in American and Korean adolescents. Journal of Korean Medical Science 201732 427-433. (https://doi. org/10.3346/jkms.2017.32.3.427)

Received in final form 30 September 2021

Accepted 11 October 2021

Accepted Manuscript published online 12 October 2021 https://ec.bioscientifica.com

https://doi.org/10.1530/EC-21-0234 (c) 2021 The authors Published by Bioscientifica Ltd
This work is licensed under a Creative Commons Attribution-NonCommercial 4.0 International License. ded from Bioscientifica.com at 04/26/2023 09:43:28AM 